
\title{
25 Research Soure \\ Clinical Utility of Neoadjuvant MRI in Early-Stage Breast Cancer Patients
}

\section{Lauren Corke}

BC Cancer Agency: British Columbia Cancer Agency

\section{Lidiya Luzhna}

The University of British Columbia

\section{Kaylie Willemsma}

The University of British Columbia

\section{Caroline IIImann}

The University of British Columbia

\section{Miranda Mcdermott}

University of Waterloo Faculty of Science

\section{Christine Wilson}

BC Cancer Agency Vancouver Centre

\section{Christine Simmons}

BC Cancer Agency: British Columbia Cancer Agency

Nathalie LeVasseur ( $\nabla$ nathalie.levasseur@bccancer.bc.ca )

BC Cancer Agency Vancouver Centre https://orcid.org/0000-0002-2581-4903

\section{Research Article}

Keywords: Breast cancer, MRI, neoadjuvant, pre-operative imaging

Posted Date: November 22nd, 2021

DOI: https://doi.org/10.21203/rs.3.rs-1062339/v1

License: (c) (i) This work is licensed under a Creative Commons Attribution 4.0 International License.

Read Full License 


\section{Abstract \\ Background}

With the increasing use of neoadjuvant treatment (NAT) for patients with early-stage breast cancer (ESBC), adequate clinical staging is essential to inform treatment. While the use of MRI with NAT has been proposed to help with accuracy of pre-treatment clinical staging, its impact in clinical practice remains controversial.

\section{Methods}

A prospective institutional database of patients with ESBC treated with NAT between May 2012 and December 2020 was analyzed in order to compare the management of patients who received an MRI prior to NAT to those who did not. The indications for MRI and correlation of MRI findings to conventional breast imaging were evaluated. The impact of MRI on management was compared between the MRI and non-MRI groups.

\section{Results}

A total of 530 patients met inclusion criteria. Of these, 186 (35.1\%) had an MRI and 344 (64.9\%) did not. The most frequent indication for MRI was the determination of disease extent (54.5\%). Patients who had an MRI prior to neoadjuvant treatment were significantly more likely to be younger (47 years versus 57 years; $p<0.001)$ and have multifocal disease $(32.3 \%$ versus $22.1 \% ; p<0.05)$. When compared to conventional imaging, MRI reported a greater extent of disease in the breast (37.6\%), more nodal involvement (18.8\%) and multifocal disease (15.1\%). Additional diagnostic interventions were advised in $52.2 \%$ of patients. who underwent MRI. Rates of mastectomies were greater in the MRI group $(80.0 \%$ versus $58.9 \% ; p<0.05)$ in addition to more axillary dissections $(28.0 \%$ versus $17.4 \% ; p<0.01)$. Rates of locoregional recurrences were low in both groups, with similar disease-free survival outcomes at 5 years.

\section{Conclusions}

MRI identified significantly more disease in contrast to conventional imaging and lead to more aggressive surgical management. Prospective studies evaluating the role of neoadjuvant MRI and its impact on long term outcomes are needed.

\section{Background}

Neoadjuvant chemotherapy is being increasingly utilized in the management of patients with early stage breast cancer [6]. While the primary objective of neoadjuvant treatment (NAT) is to achieve tumor downstaging and ideally a pathologic complete response (pCR) [3], in many cases offering neoadjuvant 
treatment can improve or increase the surgical options for a patient, especially if mastectomy is the only approach initially recommended. Adequate baseline clinical staging is of utmost importance to identify patients suitable for conversion to breast conserving surgery (BCS), to increase the chances of achieving clear margins and to inform locoregional and systemic treatment [4].

One of the modalities proposed to improve baseline clinical staging includes breast MRI, which has been extensively studied and demonstrates superior sensitivity for invasive and in situ cancer detection when compared with physical examination, ultrasonography, and mammography [19], as well as a more accurate depiction of disease extent in breast cancer patients $[5,13,19]$. MRI often detects additional areas suspicious for cancer foci [9], which, if not included in the surgical plan, increases the risk of involved margins at the time of surgery [7]. It is unclear, however, if the detection of additional disease leads to improved survival outcomes following neoadjuvant treatment $[13,19]$. While MRI is highly sensitive, it is not as specific, and its high rate of false-positive findings can lead to higher rates of additional biopsies and mastectomies [17].

Many studies have evaluated the role of preoperative MRI, with the hypothesis that its improved sensitivity may lead to more precise surgery. While some studies did in fact report lower reoperation rates in patients who received a pre-operative MRI [20], in a meta-analysis of 12 studies by Houssami et al., the impact of pre-operative MRI on surgical planning showed that approximately $11.1 \%$ of surgical alterations from the initial plan were based on true positive findings and approximately $5.5 \%$ were due to false-positive findings [9]. The use of pre-operative MRI therefore remains controversial, and there are currently no universally accepted guidelines for MRI use in the neoadjuvant setting [8]. Additionally, there are varying opinions regarding the potential use of neoadjuvant MRI, even amongst multidisciplinary expert panels (Simmons et al., 2015). In order to better understand the clinical utility of neoadjuvant MRI, we sought to understand the current use of MRI in the neoadjuvant setting in clinical practice, its correlation to conventional imaging findings and its impact on patient management.

\section{Methods}

\section{Study design}

A prospective institutional database at the British Columbia Cancer Centre in Vancouver, Canada, was interrogated to identify patients treated with neoadjuvant therapy and subsequent surgical resection between January 1, 2012 and September 30, 2020. Patients with unresectable or advanced disease at the time of presentation were excluded, as were patients who did not go on to have surgical resection. No additional exclusion criteria were applied. Database accuracy was ensured with quarterly quality assessments with in-depth audits. This project was conducted in accordance with the University of British Columbia - BC Cancer Research Ethics Board.

\section{Data collection and outcomes}


The charts of eligible patients were examined, and data was obtained from the database. The patient's demographic, clinical and pathological characteristics, imaging results, surgical outcomes and survival data were retrieved. Patients were then divided into two groups: those who received an MRI in the neoadjuvant setting, labeled the "MRI" group and those who did not, labeled the "non-MRI" group. For the MRI group, indications for MRI use, MRI findings and additional interventions following MRI were collected. MRI findings were compared to routine imaging modalities (mammography, tomosynthesis, ultrasonography) to determine if there was a discrepancy of disease extent in the breast, defined as a greater or equal to $20 \%$ difference in size based on the largest measurement in one dimension or a change in T stage based on the American Joint Committee on Cancer (AJCC) 8th Edition staging system TNM classification; a discrepancy of disease extent in the lymph nodes, defined as greater or equal to a $20 \%$ difference in size of nodal involvement or changes to nodal status (N1, N2, N3); differences in number of foci in the breast (multifocality), or multicentricity; discovery of contralateral findings; and/or additional unexpected systemic findings. Baseline surgical plans and final surgical outcomes were noted and changes were classified as "less surgery" if patients were downgraded from mastectomy to BCS or axillary dissection to sentinel node biopsy or "more surgery" if the surgical plan was changed to a mastectomy instead of BCS or axillary dissection instead of sentinel node biopsy. Intervals between time to consultation and treatment were also of interest for the MRI and non-MRI groups, as well as instances of local, regional and distant recurrences.

The primary endpoints of interest were (1) to examine the pattern of use of MRI in the neoadjuvant setting (2) to understand the informative nature of MRI in the neoadjuvant setting compared to conventional imaging modalities, and (3) to determine if MRI in the neoadjuvant setting had an impact on the management of early stage breast cancer patients treated in the neoadjuvant setting. Exploratory endpoints included the impact of MRI on time to treatment and the correlation of MRI with disease-free survival (DFS). Baseline demographic and tumor characteristics were compared between the MRI and non-MRI groups using Independent Samples t-test and Chi-Square. In all analyses, estimates were considered statistically significant for two-tailed values of $p<0.05$. The tests were carried out with SPSS 24.0 statistical software (SPSS 24.0 for Windows, 2005).

\section{Results}

\section{Patient characteristics}

A total of 530 patients who received neoadjuvant therapy between January 2012 and September 2020 were eligible for this study. Among them, 186 patients had a breast MRI prior to NAT and 344 patients did not (Table 1). The median age at diagnosis was 47 years in the MRI group and 57 in the non-MRI group ( $p$ $<0.001)$. Patients with BI-RADS breast density $C$ and D density was $32.3 \%$ in the MRI group and $22.6 \%$ in the non-MRI group ( $p=0.017)$ and was not reported in more than half of included patients. A total of $32.3 \%$ versus $22.1 \%$ had multifocal disease in the MRI and non-MRI groups $(p=0.012)$. No significant differences were noted between the grade, biomarker status, histology, pre-treatment stage or lymphovascular invasion (LVI). Rates of neoadjuvant chemotherapy use were $90.9 \%$ in the MRI group and 
83.4\% in the non-MRI group ( $\mathrm{p}=0.018$ ) as opposed to neoadjuvant endocrine therapy; a small proportion received both neoadjuvant chemotherapy and endocrine therapy, sequentially, while awaiting definitive surgical management. 
Table 1

Baseline Characteristics of patients who received an MRI in the neoadjuvant setting and those who did not.

\begin{tabular}{|c|c|c|c|}
\hline & MRI $(n=186)$ & No MRI (n=344) & $p$ value \\
\hline Age at diagnosis, y (IQR) & $47(40-55)$ & $57(49-66)$ & 0.000 \\
\hline Grade & & & 0.729 \\
\hline 1 & $14(7.5 \%)$ & $31(9.0 \%)$ & \\
\hline 2 & $80(43.0 \%)$ & $162(47.1 \%)$ & \\
\hline 3 & $77(41.4 \%)$ & $137(39.8 \%)$ & \\
\hline Unknown & $15(8.1 \%)$ & $14(4.1 \%)$ & \\
\hline ER Status & & & 0.454 \\
\hline Negative & $66(35.5 \%)$ & $134(39.0 \%)$ & \\
\hline Positive & $120(64.5 \%)$ & $210(61.0 \%)$ & \\
\hline PR Status & & & 0.122 \\
\hline Negative & $81(43.5 \%)$ & $175(50.9 \%)$ & \\
\hline Positive & 105 (56.5\%) & $169(49.1 \%)$ & \\
\hline HER2 Overall & & & 0.925 \\
\hline Negative & $117(62.9 \%)$ & $218(63.4 \%)$ & \\
\hline Positive & $69(37.1 \%)$ & $126(36.6 \%)$ & \\
\hline Clinical T & & & 0.001 \\
\hline is & $1(0.5 \%)$ & $0(0.0 \%)$ & \\
\hline 0 & $4(2.2 \%)$ & $0(0.0 \%)$ & \\
\hline 1 & $17(9.1 \%)$ & $30(8.7 \%)$ & \\
\hline 2 & $81(43.5 \%)$ & $151(43.9 \%)$ & \\
\hline 3 & $72(38.7 \%)$ & $119(34.6 \%)$ & \\
\hline 4 & $7(3.8 \%)$ & $43(12.5 \%)$ & \\
\hline
\end{tabular}

Abbreviations: $\mathrm{ER}=$ estrogen receptor; $\mathrm{PR}$ = progesterone receptor; $\mathrm{HER} 2$ = human epidermal growth factor receptor 2; IDC = invasive ductal carcinoma; ILC = invasive lobular carcinoma; IMC = invasive mammary carcinoma; DCIS = ductal carcinoma in situ; NAT = neoadjuvant treatment

*totals greater than $100 \%$ as some patients received neoadjuvant chemotherapy and endocrine therapy, sequentially 


\begin{tabular}{|c|c|c|c|}
\hline & MRI $(n=186)$ & No MRI $(n=344)$ & $p$ value \\
\hline$x$ & $2(1.1 \%)$ & $1(0.3 \%)$ & \\
\hline $1 \mathrm{mi}$ & $1(0.5 \%)$ & $0(0.0 \%)$ & \\
\hline Clinical N & & & 0.567 \\
\hline 0 & $58(31.2 \%)$ & $122(35.5 \%)$ & \\
\hline 1 & $104(55.9 \%)$ & $188(54.7 \%)$ & \\
\hline 2 & $18(9.7 \%)$ & $23(6.7 \%)$ & \\
\hline 3 & $6(3.2 \%)$ & $11(3.2 \%)$ & \\
\hline 4 & $0(0.0 \%)$ & $0(0.0 \%)$ & \\
\hline $\mathrm{X}$ & $0(0.0 \%)$ & $0(0.0 \%)$ & \\
\hline Pre-Treatment Stage & & & 0.473 \\
\hline 0 & $0(0.0 \%)$ & $0(0.0 \%)$ & \\
\hline I & $6(3.2 \%)$ & $17(4.9 \%)$ & \\
\hline ॥ & $103(55.4 \%)$ & $199(57.8 \%)$ & \\
\hline III & 77 (41.4\%) & $128(37.2 \%)$ & \\
\hline Biopsy Result & & & 0.132 \\
\hline IDC & $160(86.0 \%)$ & 306 (89.0\%) & \\
\hline ILC & $12(6.5 \%)$ & $22(6.4 \%)$ & \\
\hline IMC & $9(4.8 \%)$ & $4(1.2 \%)$ & \\
\hline DCIS & $2(1.1 \%)$ & $4(1.2 \%)$ & \\
\hline Other & $3(1.6 \%)$ & $8(2.3 \%)$ & \\
\hline Lymphovascular Invasion & & & 0.922 \\
\hline Present & $128(68.8 \%)$ & $222(64.5 \%)$ & \\
\hline Absent & $58(31.2 \%)$ & $122(35.5 \%)$ & \\
\hline BI-RADS density & & & 0.017 \\
\hline
\end{tabular}

Abbreviations: $\mathrm{ER}=$ estrogen receptor; $\mathrm{PR}=$ progesterone receptor; $\mathrm{HER} 2$ = human epidermal growth factor receptor 2; IDC = invasive ductal carcinoma; ILC = invasive lobular carcinoma; IMC = invasive mammary carcinoma; DCIS = ductal carcinoma in situ; NAT = neoadjuvant treatment

*totals greater than $100 \%$ as some patients received neoadjuvant chemotherapy and endocrine therapy, sequentially 


\begin{tabular}{|c|c|c|c|}
\hline & MRI $(n=186)$ & No MRI (n=344) & $p$ value \\
\hline A & $5(2.7 \%)$ & $9(2.6 \%)$ & \\
\hline B & $16(8.6 \%)$ & $63(18.3 \%)$ & \\
\hline C & $48(25.8 \%)$ & $60(17.4 \%)$ & \\
\hline D & $12(6.5 \%)$ & $18(5.2 \%)$ & \\
\hline Not reported & $105(56.5 \%)$ & $194(56.4 \%)$ & \\
\hline Multifocal on initial imaging & & & 0.012 \\
\hline Yes & $60(32.3 \%)$ & $76(22.1 \%)$ & \\
\hline No & $125(67.2 \%)$ & $268(77.9 \%)$ & \\
\hline Missing data & $3(1.6 \%)$ & $0(0.0 \%)$ & \\
\hline Multicentric on initial imaging & & & 0.593 \\
\hline Yes & $11(5.9 \%)$ & $26(7.65 \%)$ & \\
\hline No & $173(93.0 \%)$ & $317(92.2 \%)$ & \\
\hline Missing data & $2(1.1 \%)$ & $1(0.3 \%)$ & \\
\hline Calcifications on initial imaging & & & 0.335 \\
\hline Present & 107 (57.5\%) & $183(53.2 \%)$ & \\
\hline Absent & $45(24.2 \%)$ & $104(30.2 \%)$ & \\
\hline Missing data & $34(18.3 \%)$ & $57(16.6 \%)$ & \\
\hline NAT Chemotherapy* & & & 0.018 \\
\hline Yes & 169 (90.9\%) & $287(83.4 \%)$ & \\
\hline No & $17(9.1 \%)$ & $57(16.6 \%)$ & \\
\hline NAT Hormone therapy* & & & 0.037 \\
\hline Yes & $22(11.8 \%)$ & $65(18.9 \%)$ & \\
\hline No & $164(88.2 \%)$ & 279 (81.1\%) & \\
\hline \multicolumn{4}{|c|}{$\begin{array}{l}\text { Abbreviations: } \mathrm{ER}=\text { estrogen receptor; } \mathrm{PR}=\text { progesterone receptor; HER2 = human epidermal growth } \\
\text { factor receptor } 2 ; \mathrm{IDC}=\text { invasive ductal carcinoma; ILC = invasive lobular carcinoma; IMC = invasive } \\
\text { mammary carcinoma; DCIS = ductal carcinoma in situ; NAT = neoadjuvant treatment }\end{array}$} \\
\hline
\end{tabular}




\section{MRI Utilization and correlation to clinical and radiological findings}

The indications for breast MRI referral before NAT were reviewed. Of the 186 patients who received an MRI, 102 (54.8\%) were done to determine the extent of disease. The remaining indications were attributed to difficulty assessing the lesion on previous imaging (12.9\%), investigation of the contralateral breast (5.9\%), investigation of nodal status (4.8\%), as part of a clinical trial (1.6\%), due to young age (1.6\%), or as an annual MRI for a BRCA carrier (0.5\%). Multiple reasons were listed for 6 patients $(3.2 \%)$ and reasons were unclear in 27 cases $(14.5 \%)$.

With regard to the comparison of MRI findings to clinical examination, mammography and ultrasonography, no differences were noted between modalities in $26.9 \%$, whereas the remaining $73.1 \%$ revealed additional findings of clinical relevance with the use of MRI (Table 2). The most frequent discrepancy between MRI and other conventional imaging was a difference in the extent of disease in the breast, with $37.6 \%$ of cases showing a difference of greater than or equal to $20 \%$, all of which represented more extensive disease than what was originally reported. This resulted in an increase in tumor TNM staging in $9.7 \%$ of patients. Importantly, $15.1 \%$ of patients were found to have additional foci of involvement not previously seen on imaging and $5.4 \%$ were found to have multicentric disease which was not known prior to MRI. Additionally, $18.8 \%$ of cases revealed more extensive nodal involvement by size criteria, resulting in nodal TNM upstaging in $13.4 \%$ of patients. Amongst all patients who had an MRI, $5.4 \%$ were found to have contralateral findings requiring additional workup, of which $30 \%(3 / 10)$ were malignant findings, and $6.5 \%$ were found to have incidental systemic findings, though none of these were found to represent true metastatic disease.

Amongst the 186 patients who had an MRI, additional diagnostic interventions were advised in $52.2 \%$ of patients. These included additional mammograms for 17 patients (17.5\%), ultrasounds in 56 patients (57.7\%), core biopsies of the breast in 32 patients (33.0\%), lymph nodes in 17 patients (17.5\%) or a combined ultrasound and FNA of lymph nodes in 34 patients (35.1\%). Additional systemic imaging was requested in 10 patients $(7.2 \%)$ as a result of incidental systemic findings.

\section{Impact on patient management}

Patients in both the MRI and non-MRI groups had similar rates of change to the surgical management plan $(p=0.161)$ (Figure 1). However, if there was a change to the initial plan, it led to more extensive surgical intervention in the MRI group compared to the non-MRI group (80.0\% versus $58.9 \%$, respectively; $p<0.05)$. Within the MRI group, $80.0 \%(40 / 50)$ of patients who had a change in surgical plan went on to have more surgery in the breast, in contrast to $58.9 \%(43 / 73)$ in the non-MRI group. For those who did have more breast surgery, contralateral prophylactic mastectomy was undertaken in $56.0 \%(28 / 50)$ in the MRI group and 39.7\% (29/73) in the non-MRI group. Similarly, a change in the surgical management of the axilla was more frequent in the MRI group at $28.0 \%$ (52/186) than in the non-MRI group at $17.4 \%$ $(60 / 344)$. 
MRI did not delay the wait times for consultations and treatment. The median timeline from first biopsy to the start of neoadjuvant chemotherapy was 35 days (27-44 days) for patients who had an MRI and 32 days $(26-42$ days $)$ for those who did not receive an MRI $(p=0.166)$. Similarly, the median waiting time from Medical Oncology consultation to the start of neoadjuvant chemotherapy was 14 days (10-19 days) for those who had MRI and 13 days (10-17 days) in the non-MRI group ( $p=0.688)$. There were a total of 91 recurrences, the majority of which were distant recurrences and 68 deaths amongst all included patients. Rates of locoregional recurrences were low, occurring in 2 patients $(1.1 \%)$ for the MRI group and $7(2.0 \%)$ in the non-MRI group. With a median follow-up of 70 months, the median DFS was 30.8 months for the patients in the MRI group and 27.9 months in the non-MRI group $(p=0.579)$.

\section{Discussion}

Our study reveals that the frequency of neoadjuvant MRI was greater in younger patients with dense breast tissue and multifocal disease. In the population of patients with known early-stage breast cancer, MRI was most frequently indicated to better delineate disease extent. Additionally, our study is the first to report the clinical utility of neoadjuvant MRI, specifically with regard to how MRI aided conventional imaging in further evaluation of tumor multifocality, multicentricity, nodal involvement and contralateral breast findings. Ultimately, the identification of these findings by MRI resulted in frequent additional diagnostic interventions and more frequent alterations to surgical management plans for the breast and axilla, with higher rates of mastectomies and axillary dissections.

The fact that MRI use was more frequently used in a younger patient population is consistent with previously published data. Traditionally, MRI has been proposed as an additional screening test for younger women at high risk of breast cancer or those with a germline mutation in the BRCA1 or BRCA2 genes in whom mammography alone has lower sensitivity [12]. Additionally, MRI is more frequently recommended for younger women due to increased breast density in this patient population [26]. Given that BI-RADS has only recently been mandated for mammographic reports in BC, this information was unavailable in more than half of the patients included in this study, limiting the generalizability of these findings. Nevertheless, a higher density of breast tissue has been generally accepted as an indication for MRI [22], partially due to better fat suppression and a higher resolution [14]. This is particularly relevant prior to NAT where disease extent and accurate staging are utilized for assessment of treatment response and locoregional treatment planning [24]. Additionally, while there are no clear guidelines on who should receive an MRI in neoadjuvant settings, the most frequent reason for an MRI was to better delineate disease extent, which is consistent with earlier reports $[4-5,9,17]$. Although not adopted as a standardof-care, MRI prior to NAT is being increasingly used for the evaluation disease extent and the prediction of NAT response [24].

Our analyses demonstrated that discrepancies between clinical and radiological staging were frequent and noted in $73 \%$ of patients. To our knowledge, no prior study has directly correlated the findings between other conventional imaging, clinical extent of disease and MRI in the pre-operative setting. However, in keeping with these findings, DeMartini and colleagues summarized the results of multiple 
studies that collectively evaluated MRI in approximately 1,500 cases of newly diagnosed breast cancer. All found that MRI identified additional ipsilateral malignancy with a reported frequency that ranged from $10 \%-34 \%$ [5]. In another meta-analysis, MRI reported additional contralateral cancers in $4 \%$ of subjects [2]. One of the significant issues associated with breast MRI is the high rate of false-positive findings which overestimates the extent of the disease and can result in additional investigations, unnecessary biopsies, and more extensive surgery $[1,10]$. Our study found that just over half of the patients who underwent MRI were subjected to additional investigations. Rates of false-positive MRI findings were not evaluated as part of this study; however, the existing literature reports the false-positive rates ranging from $29-80 \%$ [10].

With regard to changes in surgical management, surgical plans for the breast and axilla were altered in nearly $27 \%$ and $28 \%$ of cases, respectively. In line with these findings, it's been previously reported that $13-$ $26 \%$ of women with invasive breast cancer have a change in surgical management based on preoperative MRI evaluation and 7-17\% alter their surgical treatment from lumpectomy to mastectomy [10]. Chen and colleagues also conducted a systematic analysis to investigate how pre- and post-NAC MRI findings affect the surgeon's recommendation [3]. They concluded that tumor size, multifocality, and disease extent on pre-treatment MRI indeed affected the initial surgical recommendation (mastectomy versus lumpectomy). However, it remains unclear that additional surgery results in lower rates of local, regional and distant relapses. While our study was not powered to detect these detect small differences, no significant differences were noted with the exploratory analyses.

Amongst the limitations, it should be noted that this was a non-randomized cohort study with a retrospective analysis of a prospective database. Additional information that was not accounted for could have impacted the frequency of changes in surgical management. Patient specific factors, such as increased anxiety [11], more extensive MRI findings than anticipated and patients seeking reconstruction for cosmesis or balancing surgery [20], may have contributed to the higher rates of change in surgical planning. We did not directly investigate if additional MRI findings and patterns of MRI use added any benefit to long-term outcomes in the studied patient population given short follow-up data, but this will be examined when longer follow-up has been established. Furthermore, a number of questions remain unanswered, including the impact of neoadjuvant MRI on the frequency of positive margins, cancer recurrence and mortality, given that data to date have not clearly established the benefits of MRI on rates of reoperation, disease recurrence, or survival [19]. To this effect, a prospectively designed trial is underway at BC Cancer in an attempt to shed light of some of these unanswered questions (NCT03790813). The results of the current study can be used to design future prospective trials to better capture the extent to which MRI findings impact intended treatment, to define the subpopulation of patients with the highest benefit from MRI, and to establish appropriate guidelines for recommending MRI prior to NAT.

\section{Conclusions}


Overall, younger patients with known multifocal disease were more likely to get an MRI referral prior to NAT. The use of neoadjuvant MRI was associated with more frequent diagnostic interventions and more extensive surgery when compared to a group without MRI use neoadjuvantly. The impact of MRI on the management of neoadjuvant patients in a prospective setting are needed, in addition to long-term followup to evaluate the impact of MRI on rates of local, regional and distant relapses and patient reported outcomes

\section{Statements And Declarations}

Funding: No funding was received for this study.

Conflict of Interest: N. LeVasseur reports receiving honoraria for participation in advisory boards from Knight, Lilly, Novartis, Pfizer, Roche, Seagen, TerSera and research funds from Abbvie and Exact Sciences. CS has received research funding from Pfizer and Amgen, and has received fees as an advisory board member from Pfizer, Amgen, Novartis, Roche, Merck, Lilly, Sandoz, and Mylan.

Ethical approval: All procedures performed in studies involving human participants were in accordance with the ethical standards of the institutional and/or national research committee and with the 1964 Helsinki declaration and its later amendments or comparable ethical standards.

Informed consent: Informed consent was not required as data was anonymized and aggregated.

\section{Declarations}

\section{Declarations}

\section{Conflict of Interest:}

N. LeVasseur reports receiving honoraria for participation in advisory boards from Knight, Lilly, Novartis, Pfizer, Roche, Seagen, TerSera and research funds from Abbvie and Exact Sciences. CS has received research funding from Pfizer and Amgen, and has received fees as an advisory board member from Pfizer, Amgen, Novartis, Roche, Merck, Lilly, Sandoz, and Mylan.

\section{Ethical approval:}

All procedures performed in studies involving human participants were in accordance with the ethical standards of the institutional and/or national research committee and with the 1964 Helsinki declaration and its later amendments or comparable ethical standards.

\section{Informed consent:}

Informed consent was not required as data was anonymized and aggregated. 


\section{Funding:}

No funding was received for this study.

\section{References}

1. Bilimoria KY, Cambic A, Hansen NM, Bethke KP (2007) Evaluating the Impact of Preoperative Breast Magnetic Resonance Imaging on the Surgical Management of Newly Diagnosed Breast Cancers. Arch Surg 142(5):441-447. https://doi.org/10.1001/archsung.142.5.441

2. Brennan ME, Houssami N, Lord S, Macaskill P, Irwig L, Dixon JM, Warren RM, Ciatto S (2009) Magnetic resonance imaging screening of the contralateral breast in women with newly diagnosed breast cancer: systematic review and meta-analysis of incremental cancer detection and impact on surgical management. J Clin Oncol 27(33):5640-5649

3. Chen JH, Feig BA, Hsiang JB, Butler JA, Mehta RS, Bahri S, Nalcioglu O, Su MY (2009) Impact of MRIEvaluated Neoadjuvant Chemotherapy Response on Change of Surgical Recommendation in Breast Cancer. Ann Surg 249(3):448-454. https://doi.org/10.1097/SLA.0b013e31819a6e01

4. Chudgar AV, Conant EF, Weinstein SP, Keller BM, Synnestvedt M, Yamartino P, McDonald ES (2017) Assessment of disease extent on contrast-enhanced MRI in breast cancer detected at digital breast tomosynthesis versus digital mammography alone. Clin Radiol 72(7):573-579

5. DeMartini W, Lehman C, Partridge S (2008) Breast MRI for cancer detection and characterization: a review of evidence-based clinical applications. Acad Radiol 15(4):408-416. https://doi.org/10.1016/j.acra.2007.11.006

6. DeMichele A, Yee D, Berry DA et al (2015) The neoadjuvant model is still the future for drug development in breast cancer. Clin Cancer Res 21(13):2911-2915. https://doi.org/10.1158/10780432.CCR-14-1760

7. Ghamrini YEI, Salama TMS, Hassan MI, Nasserb HM (2020) Mapping of multifocal breast cancer to achieve negative margins: A new step in the evolution of conservative breast surgery(A cohort study). Ann Med Surg (Lond) 56:28-33. https://doi.org/10.1016/j.amsu.2020.05.030

8. Gradishar W, Salerno KE (2016) NCCN Guidelines Update: Breast Cancer. J Natl Compr Canc Netw 14(5 Suppl):641-644

9. Houssami N, Ciatto S, Macaskill P, Lord SJ, Warren RM, Dixon JM, Irwig L (2016) Accuracy and surgical impact of magnetic resonance imaging in breast cancer staging: systematic review and meta-analysis in detection of multifocal and multicentric cancer. J Clin Oncol 26(19):3248-3258

10. Itakura K, Lessing J, Sakata T, Heinzerling A, Vriens E, Wisner D, Alvarado M, Esserman L, Ewing C, Hylton N, Hwang ES (2011) The Impact of Preoperative Magnetic Resonance Imaging on Surgical Treatment and Outcomes for Ductal Carcinoma In Situ. Clin Breast Cancer 11(1):33-38. https://doi.org/10.3816/CBC.2011.n.006 
11. LeVasseur N, Li H, Cheung W, Myers P, Mckevitt E, Warburton R, Willemsma KA, Tan AD, Chia S, Simmons C (2019) Effects of High Anxiety Scores on Surgical and Overall Treatment Plan in Patients with Breast Cancer Treated with Neoadjuvant Therapy. https://doi.org/10.1634/theoncologist.20190512. Oncologist

12. Lord SJ, Lei W, Craft P, Cawson JN, Morris I, Walleser S, Griffiths A, Parker S, Houssami N (2007) A systematic review of the effectiveness of magnetic resonance imaging (MRI) as an addition to mammography and ultrasound in screening young women at high risk of breast cancer. Eur $\mathrm{J}$ Cancer 43(13):1905-1917. https://doi.org/10.1016/j.ejca.2007.06.007

13. Mann R, Cho N, Moy L (2019) Breast MRI: State of Art. Radiology 292:520-536. https://doi.org/10.1148/radiol.2019182947

14. Marino N, Helbich T, Baltzer P, Pinker-Domenig K (2017) Multiparametric MRI of the breast: A review. Journal of Magnetic Resonance Imaging 47(2):301-315. https://doi.org/10/1002/jmri.25790

15. Masuda N, Lee S-J, Ohtani S, Im Y-H, Lee E-S, Yokota I, Kuroi K, Im S-A, Park B-W, Kim S-B, Yanagita Y, Ohno S, Takao S, Aogi K, Iwata H, Jeong J, Kim A, Park K-H, Sasano H, Ohashi Y, Toi M (2017) Adjuvant Capecitabine for Breast Cancer after Preoperative Chemotherapy. 376:2147-2159. https://doi.org/10.1056/NEJMoa. 22

16. Partridge SC, Zhang Z, Newitt DC et al (2018) Diffusion-weighted MRI Findings Predict Pathologic Response in Neoadjuvant Treatment of Breast Cancer: The ACRIN 6698 Multicenter Trial. Radiology 289(3):618-627. https://doi.org/10.1148/radiol.2018180273

17. Peters NHGM, van Esser S, van den Bosch MAAJ, Storm RK, Plaisier PW, van Dalen T, Diepstraten SCE, Weits T, Westenend PJ, Stapper G, Fernandez-Gallardo MA, Borel Rinkes IHM, van Hillegersberg R, Mali WP, Ph M, Peeters PHM (2011) Preoperative MRI and surgical management in patients with nonpalpable breast cancer: the MONET - randomised controlled trial. Eur J Cancer 47(6):879-886. https://doi.org/10.1016/j.ejca.2010.11.035

18. Pickles MD, Lowry M, Manton DJ, Gibbs P, Turnbull LW (2005) Role of dynamic contrast enhanced MRI in monitoring early response of locally advanced breast cancer to neoadjuvant chemotherapy. Breast Cancer Research and Treatment 91:1-10. https://doi-org.proxy.lib.uwaterloo.ca/ 10.1007/s10549-004-5819-2

19. Raghavendra AS, Tripathy D (2018) How Does MR Imaging Help Care for the Breast Cancer Patient? Perspective of a Medical Oncologist. Magn Reson Imaging Clin N Am 2289-293. https://doi.org/10.1016/j.mric.2017.12.013

20. Sardanelli $F$ (2010) Overview of the role of pre-operative breast MRI in the absence of evidence on patient outcomes. Breast 19(1):3-6. https://doi.org/10.1016/j.breast.2009.11.003

21. Schmitz AM, Teixeira SC, Pengel KE, Loo CE, Vogel WV, Wesseling J, Rutgers EJ, Valdés Olmos RA, Sonke GS, Rodenhuis S, Vrancken Peeters MJ, Gilhuijs KGA (2017) Monitoring tumor response to neoadjuvant chemotherapy using MRI and ${ }^{18} \mathrm{~F}$-FDG PET/CT in breast cancer subtypes. PLoS One 12(5):1-14. https://doi.org/10.1371/journal.pone.0176782 
22. Schoub PK (2018) Understanding indications and defining guidelines for breast magnetic resonance imaging. SA J Radiol 22(2):1353. https://doi.org/10.4102/sajr.v22i2.1353

23. Simmons CE, Hogeveen S, Leonard R, Rajmohan Y, Han D, Wong A, Lee J, Brackstone M, Boileau JF, Dinniwell R, Gandhi S (2015) A Canadian national expert consensus on neoadjuvant therapy for breast cancer: linking practice to evidence and beyond. Curr Oncol 22:43-53. https://doi.org/10.3747//co.22.2328

24. Sorace AG, Harvey S, Syed A, Yankeelov TE (2017) Imaging Considerations and Interprofessional Opportunities in the Care of Breast Cancer Patients in the Neoadjuvant Setting. Semin Oncol Nurs 33(4):425-439

25. Von Minckwitz G, Huang CS, Mano MS, Loibl S, Mamounas EP, Untch M, Wolmark N, Rastogi P, Schneeweiss A, Redondo A, Fischer HH, Jacot W et al (2019) Trastuzumab Emtansine for Residual Invasive HER-2 Positive Breast Cancer. N Engl J Med 380:617-628. https://doi.org/ 10.1056/NEJMoa1814017

26. Vreemann S, van Zelst JCM, Schlooz-Vries M, Bult P, Hoogerbrugge N, Karssemeijer N, Gubern-Mérida A, Mann RM (2018) The added value of mammography in different age-groups of women with and without BRCA mutation screened with breast MRI. Breast Cancer Res 20(84):1-12. https://doi.org/10.1186/s13058-018-1019-6

\section{Figures}

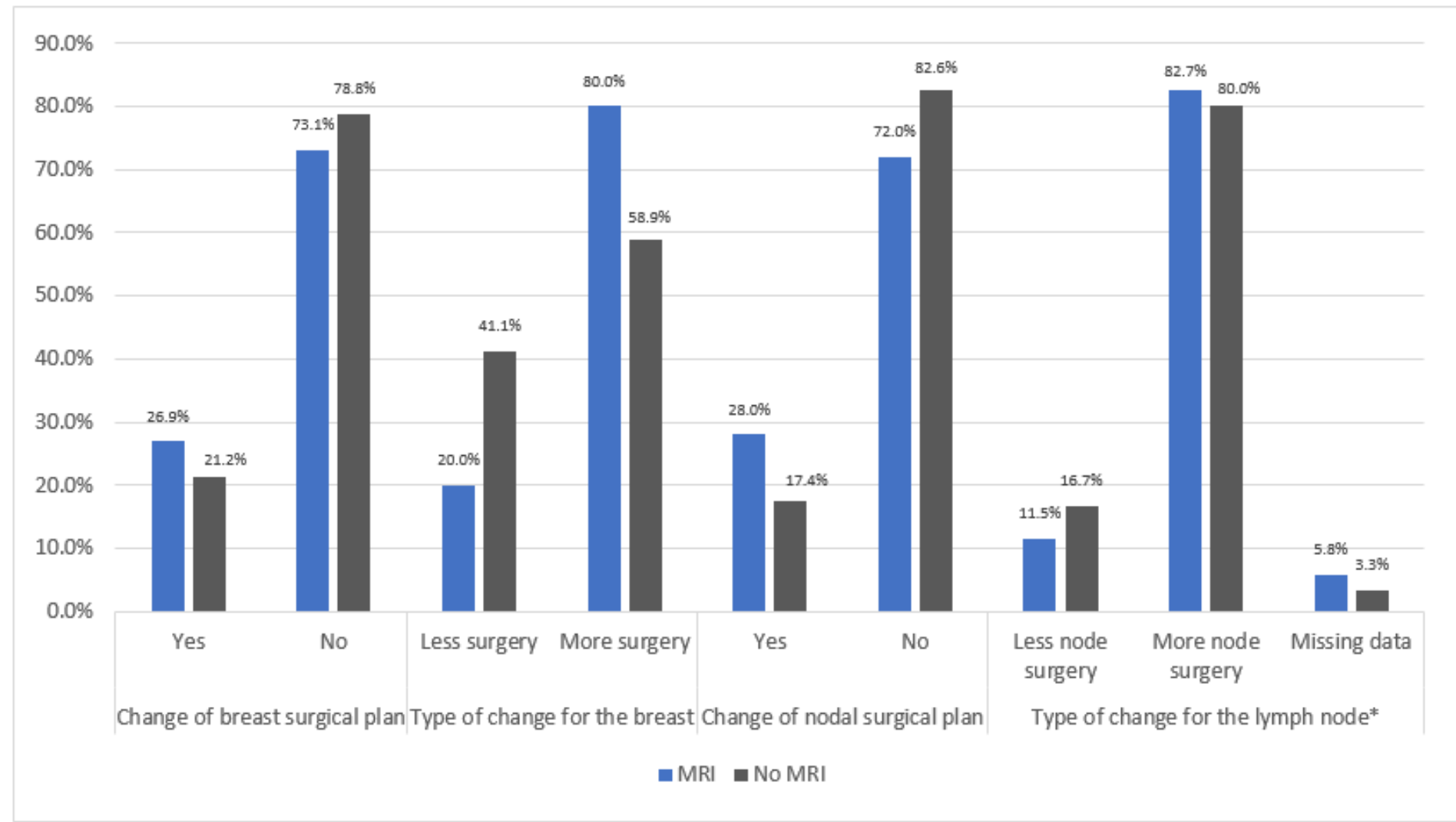

\footnotetext{
* Based on $n=52$ patients who required a change in surgical plan for the lymph nodes
} 


\section{Figure 1}

Change to the surgical management plan in the MRI and non-MRI groups, divided by breast and nodal surgical management plan 